\title{
On the Relation between Material Property and Part Performance
}

ISSN: 2576-8840

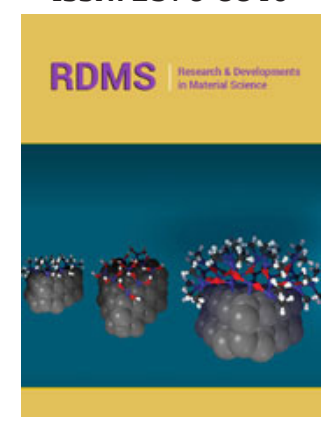

*Corresponding author: Ma Mingtu, China Automobile Engineering Research Institute, Chongqing, China

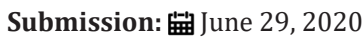

Published: 酸July 07, 2020

Volume 13 - Issue 5

How to cite this article: Ma Mingtu, Li Fuhuo, Li Hailong. On the Relation between Material Property and Part Performance. Res Dev Material Sci. 13(5). RDMS.000822. 2020. DOI: 10.31031/RDMS.2020.13.000822

Copyright@ Ma Mingtu. This article is distributed under the terms of the Creative Commons Attribution 4.0 International License, which permits unrestricted use and redistribution provided that the original author and source are credited.

\author{
Ma Mingtu ${ }^{1 *}$, Li Fuhuo ${ }^{2}$ and Li Hailong ${ }^{3}$ \\ ${ }^{1}$ China Automobile Engineering Research Institute, China \\ ${ }^{2}$ Henan Suda Electric Automobile Science and Technology Co., China \\ ${ }^{3}$ The School of Mathematics and Information, Weinan normal University, China
}

\begin{abstract}
The relation between material property and part performance is discussed. Concepts about material property and part performance are expounded. The factors influenced part performance is listed. It is of importance to deeply understand the idea about the relation between material property and part performance in practice.
\end{abstract}

Keywords: Material property; Part performance

\section{Opinion}

Material property is mechanical property including yield strength, tensile strength, elongation, reduction of area, impact toughness etc. With these parameters as the characterization of material property is good or bad. Material property is usually related to material compositions, microstructure. Parts performance refers to some related indicators which meet application requirements of parts, such as the stiffness of parts, strength, fatigue strength and fatigue life, and so on. The function of the parts usually is characterized by "performance" in English. Which is related to the properties of the material, also is related to the manufacturing process of parts. With the development of material science and technology and the deep understanding of people for the material microstructure, material properties concept and category are extended. For example, the toughness of the material is not a simple to characterize with impact value; Now many parameters i.e. The fracture toughness, dynamic fracture toughness, formability, hydrogen embrittlement and delayed fracture resistance have been introduced. Metallurgical technology property and application technology performance of materials, such as smelting, casting, rolling, welding, etc. should been also considered. The category and characterization of parts performance are extended. According to different service conditions and the environment the of parts, the characterization of parts performance is different. For example, the structural parts of the auto safety need to have the impact absorbing energy. The automobile dynamic load parts and-- power transmission system need the parameters such as fatigue and wear etc. Auto lightweight components need stiffness and the parameter of vibration and noise in the using process. For a long time, parts design is based on the basic mechanics property of the materials to evaluate the performance of the parts, but due to take different forming process, connection process of the parts, function of the final forming parts will be comprehensive effect of technological factors on the properties of materials. The predictive of parts performance is actual results of integrated influence of material properties, structure design and processing technology. So the material property is not equal to the performance of the parts. It is just based on the above concept; this paper discusses the relationship between material property and performance of parts. First, the relationships between material property and requirement of performance of parts are listed. The performance of parts under different working conditions, different stress and 
different failure modes will have different functional requirements, and therefore have different corresponding to parameter of material properties. The same property of material can achieve the different performance of parts after different processing technology or improved technology. There are a large number of examples in the automobile mechanical parts. Q\&P steel has very good toughness, the toughness of the retained austenite in the material is a good and it is also a strong hydrogen trap. The delayed fracture resistance of this material is good, but after stamping, when effective strain value is greater than some value between Ms and Md temperature. The retained austenite in the Q\&P steel will take place strain induced transformation. The retained austenite will transform to hard and brittle martensite phase. Due to becoming of toughness austenite phase to brittle martensite phase in the formed parts, so the strength and toughness of the formed parts drop sharply, also make delayed fracture resistance of parts decrease. Q\&P steel has good formability, but did not improve strength and toughness of stamping parts. The forming working will induces the secondary machining brittleness and reduces the delayed fracture resistance. Another example, shot peening can increase the surface compressive stress, improve surface structure and increase the fatigue strength of parts effectively [1]. For all kinds of spring component, the fatigue life of parts improved by shot peening is very effective. It is general difficult to improve material fatigue strength of parts, but shot peening is effective way to improve fatigue life. The welding is commonly connecting way of materials and parts; so far the related results show that the welding will reduce the performance of heat affected zone for most steel material. The strength and toughness as well as fatigue strength will drop too. But shot peening or rolling strengthening can effectively reduce the adverse effects of welding heat affected area. The rolling strengthening can effectively improve the fatigue life of the crankshaft, and other strengthening way of materials is difficult to achieve the improvement of fatigue life. These examples show that the performance of parts through the different machining way is significantly different from the property of the material.

The idea for relation between the property of material and performance of parts has a wide range of applications in the following respects. First when we carry out the reverse engineering of materials in auto Benchmark work, only base on deeply understanding the relationship between the material property and parts performance and according to the test data, we can get reliable reverse results. In order to reasonably explain secondary working embrittlement, it is necessary to that based on the relationship between the material property and parts performance, then to understand the effect of the secondary processing on the structure, property and embrittlement of materials. Database construction of material property and performance of typical parts is important demand in the development of automobile industry, but the construction of the database must have the testing data of material property and parts performance and establish the relationship between the material property and performance of typical parts. It makes database play an important role in material selection and performance prediction in design for automobile typical parts. For the gene measuring of automobile or mechanical material, we also need to correctly understand the relationship between the material property and parts performance, it can find out genetic makeup of the auto material development and application. Material production company carry out EVI services, including the application of new materials, early intervention of product design, material recommendation and performance prediction of parts, etc., the concept for relationship of the material property and parts performance is the basic gists. For the failure analysis and putting forward prevention measures of auto parts are, we must understand the material manufacturing history, the processing history of parts, service history of the parts, performance change of parts in the service process, etc.. The relationship between the material property and performance of parts is the theory of analysis tools to analyze, understand and predict of performance in forming of parts and changes of performance in the serving engineering.

Deep understanding of the relationship between the material property and performance of parts is of the very vital significance for the development of new materials, material research, the analysis of the relationship between the micro structure and material properties, as well as through the reasonable processing technology, to understand and predict the performance of the parts.

\section{Acknowledgment}

This research was supported by both National Natural Science Foundation of China (NSFC) No.U1764252 and U1760205.

\section{References}

1. Ma Mingtu (2014) The relation of material property to part function. Heat Treatment J 29(2): 1-13 in chinese. 